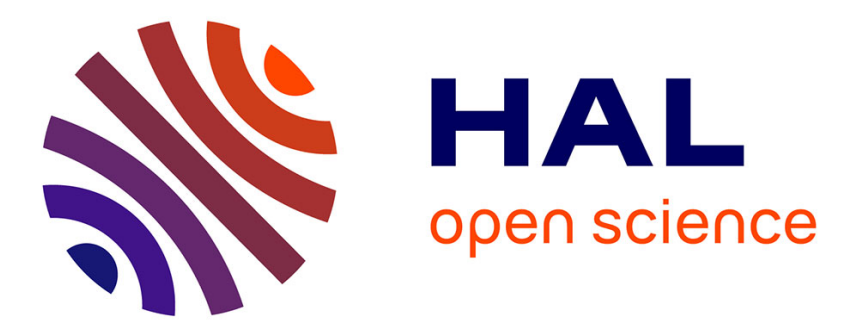

\title{
Development of colour centre lasers for spectroscopy applications
}

\author{
G. Baldacchini, G.P. Gallerano, D. Censi, M. Tonelli, P. Violino, U.M. \\ Grassano, M. Meucci, A. Scacco
}

\section{To cite this version:}

G. Baldacchini, G.P. Gallerano, D. Censi, M. Tonelli, P. Violino, et al.. Development of colour centre lasers for spectroscopy applications. Revue de Physique Appliquée, 1983, 18 (5), pp.301-306. 10.1051/rphysap:01983001805030100 . jpa-00245095

\section{HAL Id: jpa-00245095 https://hal.science/jpa-00245095}

Submitted on 1 Jan 1983

HAL is a multi-disciplinary open access archive for the deposit and dissemination of scientific research documents, whether they are published or not. The documents may come from teaching and research institutions in France or abroad, or from public or private research centers.
L'archive ouverte pluridisciplinaire HAL, est destinée au dépôt et à la diffusion de documents scientifiques de niveau recherche, publiés ou non, émanant des établissements d'enseignement et de recherche français ou étrangers, des laboratoires publics ou privés. 
Classification

Physics Abstracts

$42.55 \mathrm{R}-33.20 \mathrm{E}$

\title{
Development of colour centre lasers for spectroscopy applications
}

\author{
G. Baldacchini, G. P. Gallerano, \\ E.N.E.A. Frascati, Roma, Italy \\ D. Censi, M. Tonelli (*), P. Violino (*), \\ Istituto di Fisica, Università di Pisa, Italy \\ U. M. Grassano (*), \\ Dipartimento di Fisica, IIe Università di Roma, Italy \\ M. Meucci (*), \\ Istituto di Fisica, Università di Siena, Italy \\ and A. Scacco $\left(^{*}\right)$ \\ Istituto di Fisica, Università di Roma, Italy \\ (Reçu le 3 décembre 1982, accepté le 4 février 1983)
}

\begin{abstract}
Résumé. - On présente la description de deux lasers à centres colorés mis au point par nous-mêmes. Les détails techniques des solutions sont donnés surtout au regard de la formation des centres $F_{A}$ (II) dans les cristaux de $\mathrm{KCl}$ : Li.

Les propriétés de fluorescence de ces centres sont examinées brièvement et l'on présente un exemple d'application à la spectroscopie moléculaire.
\end{abstract}

\begin{abstract}
The description of two home-built colour centre lasers is presented. Technical details of the solutions are given with special concern to the production of the $\mathrm{F}_{\mathrm{A}}(\mathrm{II})$ centres in $\mathrm{KCl}: \mathrm{Li}$ crystals.

The fluorescence properties of these centres are briefly investigated and an example of application to molecular spectroscopy is shown.
\end{abstract}

1. Introduction. - Soon after their first realization [1] the cw colour centre lasers have attracted a considerable interest owing to their potential usefulness both in basic spectroscopic research and in applied research. At a building cost not much larger than an ordinary dye laser, they can deliver tunable narrow (single-mode) emission up to several $\mathrm{mW}$ in a spectral region (near I.R. up to and beyond $3 \mu \mathrm{m}$ ) where the only other coherent tunable continuous source is some form of semiconductor laser, with considerably lower spectral properties.

After extensive research carried out in U.S.A. and in Germany in the last few years, it is now possible, by using different kinds of colour centres, to cover almost entirely the spectral range between 0.85 and $4 \mu \mathrm{m}[2-4]$. In the $1 \mu \mathrm{m}$ region, an important application is foreseen in the field of fiber-optic communications [5], while for the whole range 1 to $4 \mu \mathrm{m}$ they have a strong spectroscopic interest : many

(*) Also : Gruppo Nazionale di Struttura della Materia del C.N.R. transitions between excited atomic levels [6-9] and many vibrational lines of organic molecules and radicals such as $\mathrm{OH}, \mathrm{HF}, \mathrm{NH}, \mathrm{CH}, \mathrm{NH}_{2}, \mathrm{CH}_{3}$ stretches [10-14], lie in this region.

In metrological applications, they may provide a link in a multiplication chain between the $10 \mu \mathrm{m}$ radiation of the stabilized $\mathrm{CO}_{2}$ laser and visible radiation by, e.g., the He-Ne laser [15]. Further spectroscopic applications can be found in the high sensitivity detection of atmospheric pollutans, realtime study of the evolution of a chemical reaction, study of decay times in the picosecond range [16], and so on.

This wide variety of possible uses has prompted several laboratories to design their own colour centre lasers, in relation to the individual needs. But the practical realization of this device involves quite a few technological problems, which have prevented up to now a wide spread diffusion of this type of laser not with standing the vast possibilities we shortly outlined above.

In our laboratories we have designed and built 
two working lasers operating in the wavelength range 2.5-2.85 $\mu \mathrm{m}$, these lasers use the same colour centre $\left(\mathrm{F}_{\mathrm{A}}(\mathrm{II})\right.$ in $\left.\mathrm{KCl}: \mathrm{Li}\right)$ but are different in design, way of optical pumping, and potential uses. In this paper we shall describe the essential features of these lasers, and how did we overcome with simple means the less common technological problems, in order to ease the way to other researchers who may wish to build their own colour centre laser for their own purpose.

2. Basic features of the laser. - The active medium of this kind of laser is an alkali-halide crystal, appropriately doped and coloured. Several colour centres have been found to lase : $F_{A}(I I), F_{B}(I I), F_{2}^{+}, F_{2}^{+}$-like and recently $\mathrm{Tl}^{\circ}$-vacancy centres $[2,3]$. Laser action has also been obtained in other host crystals [17] and, in pulsed operation only, with $F_{2}$ and $F_{2}^{-}$ centres [18]. General reviews on the colour centre lasers can be found in the literature [2,3], and therefore we shall review here only briefly the essential details that apply to our own case.

When a $\mathrm{KCl}$ crystal is heated in a potassium atmosphere, neutral lattice defects . are produced by the following mechanism. Potassium atoms stick to the surface of the crystal at the regular lattice positions where they become trapped as $\mathrm{K}^{+}$because of the thermal ionization. Chlorine ions diffuse to the surface, building new atomic planes and leaving behind anion vacancies. The electron produced by the ionization of the potassium atom become eventually trapped in the potential well produced by the anion vacancy that is electrically equivalent to a positive charge. The system vacancy + electron is called $\mathrm{F}$ centre. Upon irradiating such a centre with light of appropriate wavelength, the electron is excited into the conduction band and the vacancy is free to migrate in the crystal. However in a Li doped crystal the vacancies become easily trapped by the $\mathrm{Li}$ ions because of the positive binding energy between vacancy and impurity. As the electron recombines with the vacancy a new centre is formed : the $F_{A}$ centre [19] (see Fig. 1). The energy level diagram of this centre is also shown in the same figure together with the typical optical cycle of a four-levels laser. After optically pumping from the ground state $|\mathrm{g}\rangle$ to the excited state $|\mathrm{e}\rangle$, the system quickly $\left(\sim 10^{-12} \mathrm{~s}\right)$ relaxes to the $\mid$ re $\rangle$ state, then may radiate to the $|\mathrm{rg}\rangle$ state, that again quickly relaxes to the ground state. It is therefore relatively easy to obtain population inversion between the $\mid$ re $\rangle$ and $|\mathrm{rg}\rangle$ states, and thus laser action may start. The absorption transition $(|\mathrm{g}\rangle \rightarrow|\mathrm{e}\rangle)$ falls in the visible region while the fluorescence $(|\mathrm{re}\rangle \rightarrow|\mathrm{rg}\rangle)$ occurs in the I.R. Optical pumping may thus be accomplished by means of a fixed frequency visible laser, while infrared emission may be tuned over the fluorescence band, exactly like a dye laser.

These crystals cannot operate at room tempe-
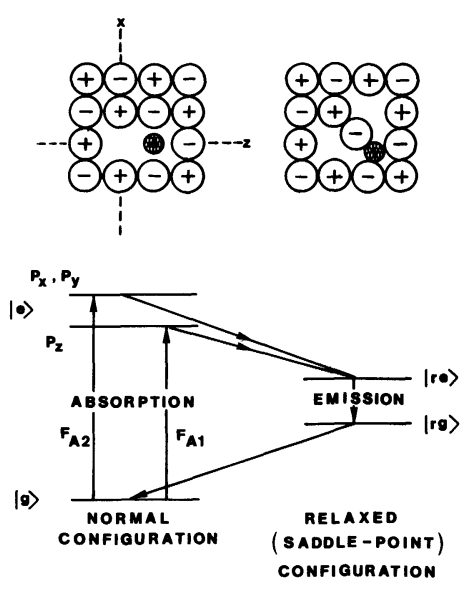

Fig. 1. - Ionic configuration and energy level diagram of the $F_{A}(I I)$ centre in the ground state (left) and in the relaxed excited state (right).

rature, since thermal motion tends to destroy $F_{A}$ centres, and also because the quantum yield of the fluorescence, which is nearly $100 \%$ at cryogenic temperature, drops to almost zero at room temperature. Lattice parameters and impurity radii allow only in a few systems (for example in $\mathrm{KCl}: \mathrm{Li}$ but not in $\mathrm{KCl}: \mathrm{Na}$ ) the relaxation of the $\mathrm{F}_{\mathrm{A}}$ centre after optical excitation, in the so called double well or saddle point configuration (Fig. 1). These centres are classified as type II or $\mathrm{F}_{\mathrm{A}}$ (II) centres and they only exhibit laser action due to their narrow emission bandwidths and short lifetimes.

3. Design and crystal preparation. - The crystal blank of $\mathrm{KCl}$ : $\mathrm{Li}$ must contain as many $\mathrm{Li}$ cations as possible in order to get a high density of $F_{A}$ (II) centres. Although our discussion will be limited here to $\mathrm{KCl}: \mathrm{Li}$, the procedure for crystal preparation will be essentially unchanged for other crystals as long as $F_{A}(I I)$ or $F_{B}(I I)$ centres are considered. Important differences exist in the case of $\mathrm{F}_{2}^{+}$and $\mathrm{F}_{2}^{+}$-like centres.

The crystal must be optically polished before coloration (formation of F-centres) takes place, because after coloration the crystal must never be exposed to green light at room temperature : only minor refinements of the polished surface can then be made under a red lamp having a negligible emission below $620 \mathrm{~nm}$; the formation of aggregate centres would otherwise be unavoidable. Since the crystal is hygroscopic and has a low hardness (Knoop 8-9 $\mathrm{kg} / \mathrm{mm}^{2}$ ), standard polishing techniques cannot be used, and some hints have recently been given by several workers $[20,21]$. After cleavage and preliminary lapping, as polishing abrasives we use alumina powders (Linde C, A, B in sequence) with reagent-grade methanol. Final crystal thickness is about $2 \mathrm{~mm}$.

After polishing, the crystal is coloured by the 
Van Doorn technique [22], with an oven modified [23] in order to gain a precise control on the $K$ vapour pressure and prevent as far as possible any damage of the polished surfaces. The coloration process lasts from 20 to $80 \mathrm{~min}$. at temperatures between 570 and $590^{\circ} \mathrm{C}$ at a vapour pressure between 1.3 and $4 \mathrm{kPa}$. Thereafter the crystal is suddenly cooled to below $200{ }^{\circ} \mathrm{C}$, in order to prevent the formation of aggregates of colour centres, by means of a flow of cool dry nitrogen, in a separate area of the oven. The crystal is checked and it is thereafter loaded into the laser cryostat (to be described below), cooled to $-30^{\circ} \mathrm{C}$ and irradiated with green light (from a tungsten lamp with a filter) for $30 \mathrm{~min}$. With this process we obtain the aggregation of $\mathrm{F}$ centres and $\mathrm{Li}$ impurities, giving rise to the $F_{A}($ II $)$ centres as described above. At $-30^{\circ} \mathrm{C}$ the ion mobility is still high enough for the aggregation to occur, without risk of getting more complex aggregate that would absorb laser and pump radiation. After the aggregation is complete, the crystal is cooled to liquid nitrogen temperature, where the ion mobility is negligible. At this temperature the colour centres are stable even under the strong optical pumping necessary for the laser action.

4. Mechanical set-up. - The optical cavity is a folded, astigmatically compensated cavity [24] of the type commonly used in $\mathrm{cw}$ dye lasers. The mechanical set-up of the mirrors is of course complicated by the need to work at cryogenic temperature and therefore under vacuum.

The theory of the optical cavity has been given in reference [24]; we shall therefore not give here the details of its design : the dimensions have been calculated by keeping in mind the crystal size, the required mode spacing and the stability range of the system. Details of this computations can be found in reference [25]. The most important difference between the two lasers we built is the method of pumping. In one case (in Frascati, Roma) the source was an $\mathrm{Ar}^{+}$laser, and we decided to pump the colour centre laser through the end mirror of the optical cavity. This mirror has a dichroic coating transmitting the $\mathrm{Ar}^{+}$green radiation and reflecting the infrared radiation. A general view of this laser is shown in figure 2. The end mirror and the folding mirror are inside the cryostat; the optical alignment must therefore be carried out at room temperature with a dummy crystal in place of the coloured crystal. After the cryostat has been evacuated, the only external adjustments are those of the output coupler and the lens $L_{2}$ for properly focussing the pump light to the mode waist of the laser. This approach is very simple and gave satisfactory results ; it requires however a rather uncommon dichroic coating for the end mirror. The schematic of the optical cavity is shown in figure 3 with details of the output coupling and tuning.

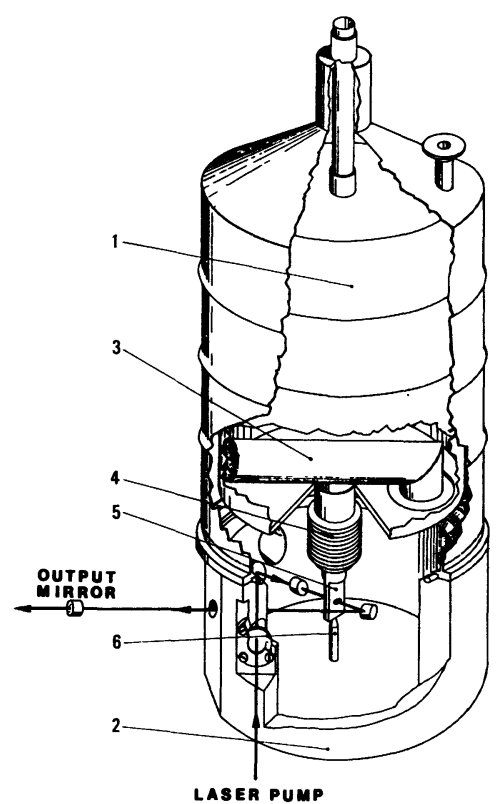

Fig. 2. - Schematic of the Frascati-Rome laser : 1) Liquid nitrogen container, 2) Optical cavity vacuum enclosure, 3) Cryogenic vacuum pump, 4) Metallic bellows, 5) Cold finger with crystal holder, 6) Insulating support.

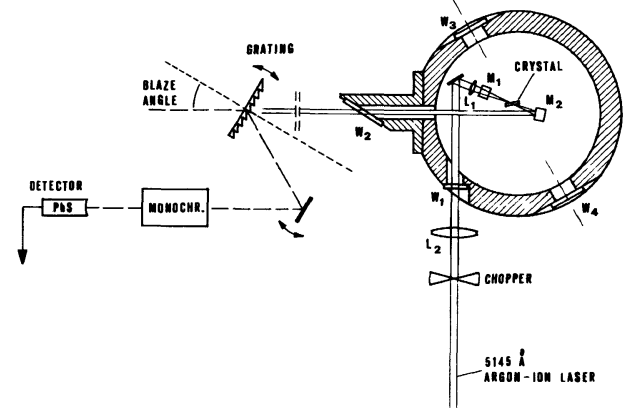

Fig. 3. - Schematic of the laser cavity and tuning arrangement of the Frascati-Rome laser with pumping through the dichroic mirror $M_{1}, M_{2}$ is the folding mirror, $w_{1}$ and $w_{2}$ the pumping and the coupling windows, $L_{1}$ and $L_{2}$ are the focussing lenses. The auxiliary windows $w_{3}$ and $w_{4}$ are used for the aggregation of the $F$ centres in order to form the $F_{A}($ II) centres.

In the other laser (in Pisa) the pump source was a $\mathrm{Kr}^{+}$laser, and we decided to avoid the dichroic mirror by pumping the crystal through an uncoated $\mathrm{ZnSe}$ beamsplitter, located between the folding mirror and the output coupler (see Fig. 4). It is positioned at Brewster's angle for the IR radiation, and it reflects about $50 \%$ of the visible, vertically polarized, radiation. This arrangement has the advantage of being usable without modifications with several pump sources and with several active crystals, since it is essentially wavelength independent, the end mirror and the folding mirror both having a broadband metallic coating. The positioning of all mirrors is accessible under vacuum by means of appropriate 


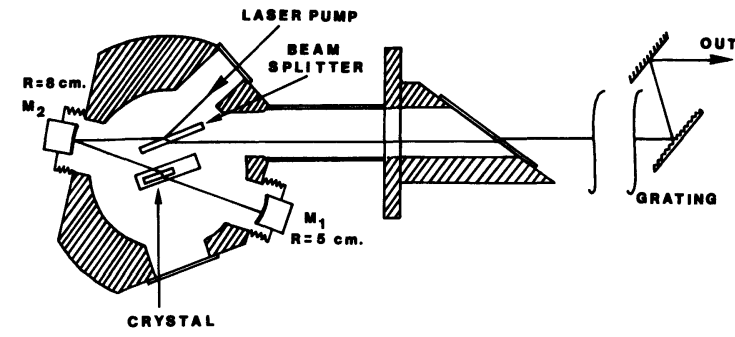

Fig. 4. - Schematic of the laser cavity and tuning arrangement of the PISA laser with pumping through a beam splitter.

bellows. Fine adjustment of the pump focussing is accomplished by changing the input beam direction. In addition, in order to limit power fluctuations due mechanical instabilities, all mirrors have been fastened to a single superinvar plate, whose size is $115 \times 17 \times 1.5 \mathrm{~cm}$.

In both lasers the output coupler is outside the vacuum chamber, and with this kind of mounting the output mirror can be replaced by a tuning element (grating or prism plus mirror) and an absorption cell can be easily placed intracavity. Because of the atmospheric water vapour absorption around $2.8 \mu \mathrm{m}$, the region between the $\mathrm{CaF}_{2}$ window and the output mirror is sealed in an airtight enclosure flushed with dry nitrogen.

5. Fluorescence measurements. - There is an optimum for the density of $F$ centres in the crystal. If their number is small, not enough $F_{A}$ (II) centres are formed during the aggregation process in order to absorb most of the pump light, thus increasing the effective threshold. If there are too many $F$ centres, not all of them will form $F_{A}$ (II) centres, and the remaining $F$ centres will absorb uselessly pump radiation, and might even absorb infrared radiation. In addition, in an optically thick crystal, part of the crystal has no useful purpose and rather increases the threshold. It is however difficult to state precisely the optimum $\mathrm{F}$ centre density, and only vague indications about it are given in the literature. Therefore we decided to measure, under the same conditions, the fluorescence from the $F_{A}$ (II) centres of variously coloured crystals with the assumption that the crystals that give the highest fluorescence are those with the highest visible absorption and the lowest infrared sèlf-absorption.

The results are given in figure $5:$ the fluorescence intensity normalized to the incident power is plotted versus the measured $F_{A}$ centres density. Two sets of results are given for vertical and horizontal polarizations of the pump. A maximum can clearly be identified, and experiments showed that the crystal with the $F_{A}$ centres density near this maximum consistently had lower threshold than the other ones. This is therefore an easy way for identifying the most likely candidates for lasing with low thresholds. The lumi-

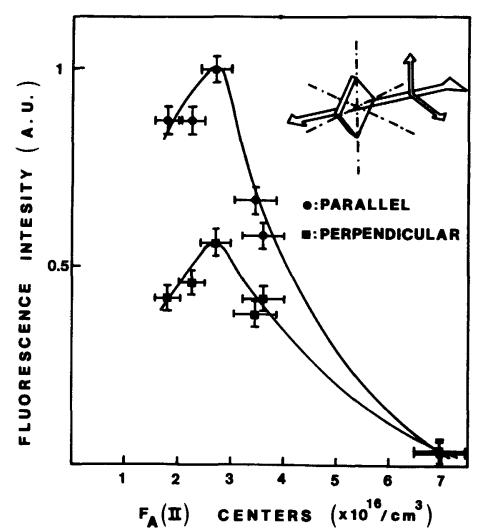

Fig. 5. - Fluorescence intensity as a function of the $F_{A}($ II $)$ centres concentration. The pumping beam $(\lambda=6471 \AA)$ is polarized parallel or perpendicular to the incidence plane.

nescence decrease at high $F_{A}$ centres concentration is probably analogous to the quenching of the $F$ centre emission found by Miehlich [26], but it occurs at much lower centres concentration.

Of considerable significance is the difference between the two sets of data with different pump polarization. The crystal is installed into the cavity at Brewster's angle and with the $\langle 110\rangle$ axis vertical. The $F_{A}$ centre axis (anion vacancy-cation impurity direction) can be oriented along all three crystal axes, but in the absorption process the dipole moments of the transitions depend upon the direction of the centre and the polarization of the light. The high energy band in the green $\left(\mathrm{F}_{\mathrm{A} 2}\right.$ band in Fig. 1) absorbs light polarized perpendicularly to the centre axis, while the lower energy band in the red $\left(F_{A 1}\right.$ band) absorbs light polarized parallel to the centre axis.

Moreover, after excitation, the centre can change its orientation due to a jumping of the vacancy around the impurity during the relaxation process.

Therefore if one pumps the system with polarized light, reorientation of the centres (along directions such that they can no longer be excited), leads to a progressive decrease of the absorption and the system becomes transparent to the pumping light. With a crystal oriented at Brewster angle to the incident pumping beam, all centres absorb horizontally polarized light in the $\mathrm{F}_{\mathrm{A} 2}$ band (green) thus preventing the alignment of the centres in a particular direction. On the other hand, on pumping in the $F_{A 1}$ band (red) with a vertically polarized beam one could align the centres and bleach the absorption if the "vertical direction " coincides with the $\langle 001\rangle$ direction of the crystal.

Consequently there are no problems if one uses, like in the Rome-Frascati laser, a horizontally polarized pump through the end mirror. However in a laser that is pumped by means of a beamsplitter, like the Pisa laser, where it is essential to use a vertical polarization (unless the beamsplitter has very excep- 
tional properties), one has to take care of the alignment of the crystal in order to avoid bleaching. Therefore it was important to test in this case whether the fluorescence efficiency with vertically polarized pump (at $\lambda=6471 \AA$ ) was comparable to that with horizontal polarization. Figure 5 shows that with $\mathrm{KCl}: \mathrm{Li}$ at $77 \mathrm{~K}$ the two fluorescence intensities are comparable, and that one can therefore use the uncoated beamsplitter without too large a loss.

6. Wavelength tuning. - In order to change the emission wavelength within the fluorescence band, we used both a prism [27] and a grating [28]. As a prism we use a $60^{\circ}$ sapphire prism, whose surfaces lie almost exactly at Brewster's angle when the prism is placed in the symmetrical position; tuning can be accomplished by rotating the output mirror around a vertical axis. As a grating we use a Bausch \& Lomb replica with 420 grooves $/ \mathrm{mm}$, blazed at $2.15 \mu \mathrm{m}$, in a Littrow mount, the zeroth order reflection being the output beam; tuning is accomplished by rotating the grating. We use a further mirror in front of the grating and parallel to it (see Fig. 4) to steer the output beam. Both this mirror and the grating are mounted on a same rotatory stage, so that, on changing the wavelength, the output beam is neither translated nor changed in direction.

Both prism and grating work satisfactory, but operation of the grating proved to be easier. In order to operate the laser in a single mode, we use an intracavity etalon with special coating; this etalon has a low finesse (about 2) but very low losses in the infrared.

7. Laser performances. - At the threshold the pumping power reaching the crystal was about $100 \mathrm{~mW}$ for the free-running laser equipped with a $95 \%$ reflectivity output mirror. With ageing, the lasing threshold increases (Fig. 6) but fairly slowly, and all crystals were still usable one month after preparation.

With the grating as a tuning element, the threshold increases to $230 \mathrm{~mW}$ at the emission peak, with dry nitrogen flushing the arm outside the vacuum chamber. The tunability range is from 2.50 to $2.85 \mu \mathrm{m}$ in the absence of water vapour absorption. The maximum power is about $4 \mathrm{~mW}$ with $850 \mathrm{~mW}$ pump power on the crystal from the $\mathrm{Kr}^{+}$laser, and the overall emission width is about $10 \mathrm{GHz}$ (without the mode selecting etalon).

Even though the work on the lasers is still in progress to reduce line-width and increase the spectral range

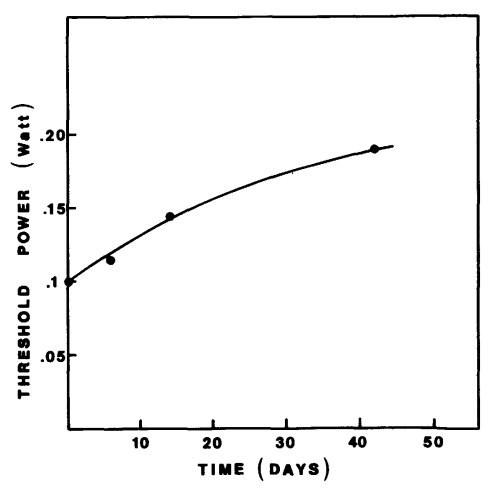

Fig. 6. - Increase of the laser threshold of the colour centre laser as function of ageing time.

by use of other crystals, we already obtained some spectra that show usefulness of this kind of source in molecular spectroscopy. A spectrum of water has been obtained intracavity and spectra of $\mathrm{CH}_{3} \mathrm{OH}, \mathrm{HCOOH}$ and $\mathrm{CH}_{3} \mathrm{COOH}$ have been studied by means of optoacoustic detection with the apparatus described in reference [29]. The results of these investigations will be discussed in a forthcoming paper; a spectrum is shown in figure 7, just as an example of the versatility and capabilities of these sources.

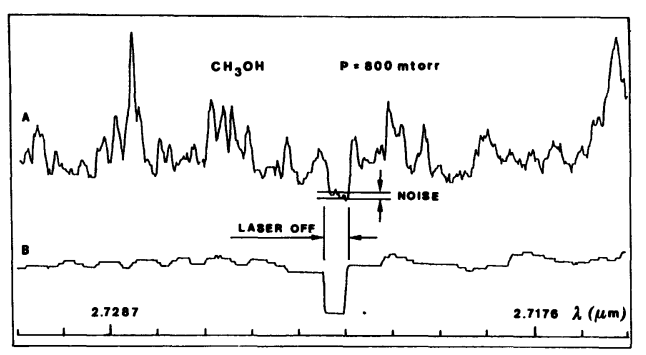

Fig. 7. - Optoacoustic spectrum of $\mathrm{CH}_{3} \mathrm{OH}$ molecules (A) and laser output (B). The amount of noise can be deduced from the interval at the centre of the spectrum where the laser was blanked.

Acknowledgments. - The authors would like to thank warmly Dr. L. F. Mollenauer and Dr. G. Litfin for their precious advices and help in the first stage of construction of our lasers and Dr. P. Minguzzi for stimulating discussions during this work.

The technical help of P. Cardoni, I. Cenciarelli, L. Mori, M. Montanari and A. Serafini is gratefully acknowledged.

\section{References}

[1] Mollenauer, L. F. and Olson, D. H., Appl. Phys. Lett. 24 (1974) 386.

[2] Mollenauer, L. F., Methods of Experimental Physics vol. 15B, edited by C. L. Tang (Academic Press, London), 1979, p. 1.
[3] Gellerman, W., Koch, K. P., Lüty, F., Laser Focus $18 \mathrm{n}^{\circ} 4$ (1982) 71.

[4] Schneider, I., Opt. Lett. 7 (1981) 271.

[5] Mollenauer, L. F., Stolen, R. H., Gordon, J. P., Phys. Rev. Lett. 45 (1980) 1095. 
[6] Nacher, P. J., Leduc, M., Trénec, G., Lalö̈, F., J. Physique Lett. 43 (1982) L-525.

[7] Begemann, M. H., Saykally, R. J., Opt. Commun. 40 (1982) 277.

[8] Gerhardt, H., Hansh, T. W., Opt. Commun. 41 (1982) 17

[9] JACKSON, D. J., ARimondo, E., LAWLer, J. E., HANSH, T. W., Opt. Commun. 33 (1980) 51.

[10] Beigang, R., Litfin, G., Schneider, R., Phys. Rev. A 20 (1979) 229.

[11] Litfin, G., Pollok, G. R., Curl, R. F., Tittel, K., J. Chem. Phys. 72 (1980) 6602.

[12] Pfeiffer, J., Kirsten, D., Kalkert, P., Urban, W., Appl. Phys. B 26 (1981) 173.

[13] Gough, T. E., Scoles, G., Laser Spectroscopy V, edited by McKellar A. R. W., Oka T., Stoicheff B. P. (Springer-Verlag, Heidelberg), 1981, p. 337.

[14] German, K. R., Gornell, W. S., J. Opt. Soc. Am. 71 (1981) 1452.

[15] Evenson, K. M., Laser Focus 17 no 11 (1981) 61.

[16] Wiesenfeld, J. M., Mollenauer, L. F., IPPEN, E. P., Phys. Rev. Lett. 47 (1981) 1668.
[17] Henderson, B., Opt. Lett. 6 (1981) 437.

[18] Gusev, Yu. L., Marennikov, S. I., Chebotayev, V. P., Sov. Tech. Phys. Lett. 3 (1977) 124.

[19] Lüty, F., Physics of Color Centers, edited by Fowler W. B. (Academic Press London) 1968, p. 181.

[20] Fuchs, B. A., Appl. Opt. 18 (1979) 1125.

[21] Merdes, D. W., Appl. Opt. 18 (1979) 4063.

[22] VAN Doorn, C. Z., Rev. Sci. Instrum. 32 (1961) 755.

[23] Mollenauer, L. F., Rev. Sci. Instrum. 49 (1978) 809.

[24] Kogelnik, H. W., Ippen, E. P., Dienes, A., Shank, C. V., IEEE J. Quantum Electron. 8 (1972) 373.

[25] Baldacchini, G., Gallerano, G. P., Grassano, U. M., Musone, A., Violino, P., Meucci, M., C.N.E.N. report 80.13 (Centro Frascati, Rome) 1980.

[26] Miehlich, A., Z. Phys. 176 (1963) 168.

[27] Beigang, R., Litfin, G., Welling, H., Opt. Commun. 22 (1977) 269.

[28] German, K. R., Opt. Lett. 4 (1979) 68.

[29] Di Lieto, A., Minguzzi, P., Tonelli, M., Opt. Comm. 31 (1979) 25. 